\title{
COVID-19 Infection Presenting as Acute Onset Focal Status Epilepticus in a Nine Year Old Boy
}

\author{
Jeffrey A. Swarz, Sarah Daily, Emily Niemi, Samuel G. Hilbert, Hala Ali Ibrahim, Daniel A. Rauch and John N. Gaitanis* \\ Tufts Floating Hospital for Children, Tufts Medical Center, Boston, Massachusetts, USA
}

${ }^{\star}$ Corresponding author: John Gaitanis, Pediatric Neurology, Department of Pediatrics, Tufts Floating Hospital for Children, 800 Washington Street, Boston, MA, 02111, USA; Tel: 617-636-5356; Fax: 617-636-8375; E-mail: jgaitanis@tuftsmedicalcenter.org

Received: August 10, 2020; Accepted: August 18, 2020; Published: August 24, 2020

\begin{abstract}
Atypical presentations of COVID-19 in children, such as new onset seizures must be recognized, and warrant liberal testing to prevent spread of the disease. We describe the unique presentation of a child with acute onset focal status epilepticus and vomiting, positive for COVID-19. Patient demographics, history, neurological findings, MRI, treatment, and prognosis were reviewed. The literature was reviewed for prior case reports. This nine year old boy had an episode of vomiting followed by acute onset focal status epilepticus. He was able to walk with assistance but could not speak or follow most commands. He had persistent left eye gaze deviation which could not be overcome and loss of motor function in his right arm. He also developed left arm automatisms. These symptoms lasted for approximately 90 minutes before resolution following administration of lorazepam. Ten hours after the onset of symptoms our patient developed a temperature of 38.6 and began vomiting again. COVID-19 testing was performed and resulted positive. He had no medical history, no known sick contacts, and there was no family history of seizures or epilepsy. Pediatric patients with COVID-19 present with a broader spectrum of symptoms than adults. While fever and cough are the most common presenting symptom in both the pediatric and adult populations, GI symptoms are more common in the pediatric population. It is important to be aware that atypical presentations including neurologic symptoms are being noted in the pediatric population and may indicate the presence of infection rather than another etiology. This is only the second documented pediatric patient in the world to present with seizure and test positive for COVID-19.
\end{abstract}

Keywords: Children, Novel Corona virus, Status epilepticus

\section{Introduction}

Estimates suggest children currently account for $1 \%-5 \%$ of diagnosed COVID-19 cases [1,2]. Available literature focusing on children is limited in comparison to adults but suggests the clinical spectrum of illness in the pediatric population associated with severe acute respiratory syndrome coronavirus 2 (SARS-CoV-2) which causes coronavirus disease 2019 (COVID-19) is distinct from and broader than that in the adults. The most frequently reported symptoms in adults with COVID-19 include fever, dry cough, fatigue, and in severe cases dyspnea is common [3]. Children, when symptomatic, also present with fever and cough, but a significant proportion present with atypical symptoms such as vomiting and diarrhea [4,5]. A large proportion of COVID-19 positive children are asymptomatic or have mild cases of disease [6,7]. This difference in presentation and severity may lead to underdiagnosis in children facilitating spread of the disease [8]. This report documents the first reported case of a COVID-19 positive child presenting with focal status epilepticus.

\section{Case History}

On the evening of admission our patient, a healthy 9 year old boy of Brazilian Portuguese descent, was at his aunt's home for dinner. At 2200 hours he became nauseous and vomited one time. He was given an antacid and driven back to his parents' house where he arrived at $2300 \mathrm{hrs}$. He required assistance but was able to walk into his house where his mother noticed fixed eye deviation to the left, inability to move the right arm, and was unable to speak. He was able to follow commands and was even able to take a shower. One hour after symptom onset, he was reaching out with his left hand for objects that were not there. He was also producing clicking sounds with his tongue. His mother became concerned that he was not improving and transported him to the Emergency Department for evaluation where he was able to walk under his own power.

In the Emergency Department, he was afebrile and vital signs were normal for age. There were no signs of meningismus. He had fixed left eye deviation with absent oculocephalic reflexes, $4 \mathrm{~mm}$ pupils responsive bilaterally, a fine tremor in the distal right upper extremity, which was not suppressible, and left arm automatisms. He was aphasic. Our patient was given $1 \mathrm{mg}$ lorazepam IV which terminated his gaze deviation, abnormal movements, and aphasia with return to baseline mental status shortly afterwards. He was then loaded with levetiracetam $20 \mathrm{mg} / \mathrm{kg}$. This child had no significant medical history, immunizations were up to date, took no medications, had no drug allergies, had no history of hospitalizations or surgeries, and had no family history of seizures or epilepsy. He had no recent head trauma, no fevers, URI symptoms, GI symptoms, sick contacts, or recent foreign travel. 


\section{Clinical Findings}

Laboratory evaluations included a glucose level of $147 \mathrm{mg} / \mathrm{dl}$. CBC and Chem 10 were normal. LFTs showed a mild transaminitis. Urine toxicology screen and chest $\mathrm{x}$-ray were normal. CT of the head without contrast was normal. LTM vEEG was abnormal due to the presence of nearly continuous delta slowing seen broadly throughout the right hemisphere, indicative of cortical and subcortical dysfunction. No epileptiform features were seen. Following admission overnight, our patient had returned to baseline mental status without any further clinical seizure activity. On the morning following admission, ten hours after his initial onset of symptoms, our patient developed a temperature of $38.6 \mathrm{C}$ and experienced multiple episodes of vomiting. He was given ondansetron for nausea and acetaminophen for fever, after which symptoms resolved. In the setting of the COVID-19 pandemic, the acute onset of fever with GI symptoms suggested possible infection with the coronavirus. A test was obtained and resulted positive later on the day of admission. An MRI of the Brain was planned due to the focal neurological findings but was deferred due to his positive coronavirus test. Due to the highly infectious nature of COVID-19, his MRI would require scheduling during a low volume period with additional time required to disinfect the scanning equipment, in turn prolonging his admission and possibly exposing staff and other patients. Our patient was asymptomatic and had a normal examination. Thus the decision was made to provide pharmacologic coverage for seizure control and discharge to home, with outpatient MRI follow up after quarantine.

Our patient was in optimal health prior to the onset of symptoms. His presentation with acute focal status epilepticus and vomiting was typically concerning for an infectious etiology, intracranial process, or structural abnormality. He was afebrile, without leukocytosis, had no signs of meningismus or encephalopathy, and had no known sick contacts, so a lumbar puncture was deferred and antibiotics were not started. Computed tomography of the head was normal, indicating mass or hemorrhages were unlikely. Resolution of his presenting symptoms with lorazepam suggested seizure as an etiology.

\section{Discussion}

Prior to the COVID-19 result, thought was also given to norovirus and rotavirus infections as those two viruses are associated with seizures in children. Benign convulsions associated with mild gastroenteritis (CWG) are associated with norovirus and rotavirus, however, the highest incidence occurs in children 12-24 months, and is characterized by generalized tonic-clonic seizure activity lasting $<5$ minutes [9]. This was inconsistent with our patient's presentation. Our patient was outside of the expected age group for febrile seizures or CWG, and his presenting seizure was prolonged and focal in semiology. Furthermore, his electroencephalogram the following day revealed diffuse and continuous right hemispheric slowing. All of these features were atypical for febrile seizures or CWG. Yet, given his rapid return to baseline neurological functioning the following morning, acute infectious encephalitis and stroke were similarly unlikely.

Our patient was discharged to home quarantine. At his follow up visit two weeks later, our patient reported no further seizure activity and had returned to his baseline excellent health. Magnetic resonance imaging of the brain was normal without any signs for ischemic injury or structural lesions to account for his seizure. Anticonvulsant therapy was continued pending a following EEG. The current COVID-19 literature documents the clinical symptoms of over 3000 children world-wide and the spectrum of disease in children is still emerging. Only one two year-old girl in China has previously been reported to present with fever, convulsions, and GI symptoms and test positive for COVID-19 [10]. By contrast, the adult literature contains a growing number of COVID-19 positive patients presenting with central nervous system symptoms, including encephalopathy and seizures. A recent report describes a 78 year old woman presenting with focal status epilepticus as a unique clinical feature of COVID-19 [11]. While the link between COVID-19 infection and epileptogenesis has not been established yet, there is a physiologically logical hypothesis to propose linking the two. First, infection is by far the leading cause of focal status epilepticus in children, even outside the setting of direct infection of the meninges. Coronavirus is also thought to cause a dysregulated immune response which ultimately causes much of the morbidity and mortality associated with the disease. It is reasonable that an infectious etiology such as COVID-19 may cause seizures through alterations of cytokine responses, which are also implicated as an etiologic factor in febrile seizures. In this time of pandemic, it is important to recognize atypical presenting symptoms of COVID-19 such as new onset seizures, test liberally, and isolate those infected to prevent spread of the disease [12]. If focal status epilepticus is a unique presenting feature of COVID-19, our patients' full outcome suggests that this rare presentation may portend a favorable prognosis.

\section{Competing Interests}

The authors declare that they have no competing interests.

\section{Funding Information}

No internal or external funding for this manuscript. The authors have indicated they have no financial relationships relevant to this article to disclose.

\section{References}

1. Tezer H, Bedir Demirdağ T (2020) Novel coronavirus disease (COVID-19) in children. Turk J Med Sci 50: 592-603. [crossref]

2. Ludvigsson JF (2020) Systematic review of COVID-19 in children shows milder cases and a better prognosis than adults. Acta Paediatr 109: 1088-1095. [crossref]

3. Rothan HA, Byrareddy SN (2020) The epidemiology and pathogenesis of coronavirus disease (COVID-19) outbreak. J Autoimmun 109: 102433. [crossref]

4. Cai J, Xu J, Lin D, Zhi Yang, Lei Xu, et al. (2020) A Case Series of children with 2019 novel coronavirus infection: clinical and epidemiological features [published online ahead of print, $2020 \mathrm{Feb} 28$ ]. Clin Infect Dis ciaa198. [crossref]

5. Hong H, Wang Y, Chung HT, Chen CJ (2020) Clinical characteristics of nove coronavirus disease 2019 (COVID-19) in newborns, infants and children. Pediatr Neonatol 61: 131-132. [crossref]

6. Cruz AT, Zeichner SL (2020) COVID-19 in Children: Initial Characterization of the Pediatric Disease. Pediatrics e20200834. [crossref]

7. Tan YP, Tan BY, Pan J, Wu J, Zeng SZ, et al. (2020) Epidemiologic and clinical characteristics of 10 children with coronavirus disease 2019 in Changsha, China. J ClinVirol 127: 104353. [crossref]

8. Chang TH, Wu JL, Chang LY (2020) Clinical characteristics and diagnostic challenges of pediatric COVID-19: A systematic review and meta-analysis. J Formos Med Assoc 119: 982-989. [crossref] 
John N. Gaitanis (2020) COVID-19 Infection Presenting as Acute Onset Focal Status Epilepticus in a Nine Year Old Boy.

9. Ma X, Luan S, Zhao Y, Lv X, Zhang R (2019) Clinical characteristics and follow-up of benign convulsions with mild gastroenteritis among children. Medicine (Baltimore) 98: e14082. [crossref]

10. Tan YP, Tan BY, Pan J, Wu J, Zeng SZ, et al. (2020) Epidemiologic and clinical characteristics of 10 children with coronavirus disease 2019 in Changsha, China. $J$ ClinVirol 127: 104353. [crossref]
11. Vollono C, Rollo E, Romozzi M, Frisullo G, Servidei S, et al. (2020) Focal status epilepticus as unique clinical feature of COVID-19. a case report, Seizure: European Journal of Epilepsy 78: 109-112. [crossref]

12. Shen K, Yang Y, Wang T, Dongchi Zhao, Yi Jiang, et al. (2020) Diagnosis, treatment, and prevention of 2019 novel coronavirus infection in children: experts' consensus statement. World J Pediatr 16: 223-231. [crossref]

\section{Citation:}

Swarz JA, Daily S, Niemi E, Hilbert SG, Ibrahim HA, Rauch DA and Gaitanis JN (2020) COVID-19 Infection Presenting as Acute Onset Focal Status Epilepticus in a Nine Year Old Boy. J Neurol Neurocrit Care Volume 3(2): 1-3. 